\title{
Metaplastic breast carcinoma: a rare entity
}

\author{
Nickos G. Kelessis • George D. Plataniotis • \\ Manolis V. Agapitos • Georgia N. Ioanidou • \\ Irene T. Georgiou • Tina E. Coclami
}

Received: 5 October 2009/Accepted: 20 November 2009/Published online: 18 December 2009

(C) Springer-Verlag 2009

\begin{abstract}
Metaplastic breast carcinoma represents a very rare neoplasm which accounts for less than $1 \%$ of all breast malignancies. The tumour usually consists of epithelial and non-epithelial elements in different amounts and variables. Herein, we present a case of a 59-year-old woman who presented with a 7-month history of a large painless mass in the upper outer quadrant of her left breast that proved to be a metaplastic carcinoma. The metastatic workup revealed one small metastasis in the upper lobe of the right lung. Treatment included neoadjuvant chemotherapy, surgery,
\end{abstract}

N. G. Kelessis

Department of General Surgery, Neo Athineon Gen Hospital, Athens, Greece

G. D. Plataniotis

1st Surgical Oncology Clinic, St Savvas Anticancer Institute,

Athens, Greece

M. V. Agapitos

Laiko University Hospital,

Athens, Greece

G. N. Ioanidou

Radiotherapy Department, St Savvas Anticancer Institute,

Athens, Greece

\section{T. Georgiou}

Radiology Department, St Savvas Anticancer Institute,

Athens, Greece

T. E. Coclami

PhD City University,

London, UK

N. G. Kelessis $(\bowtie)$

43 Metaxa str. Glyfada,

16674 Athens, Greece

e-mail: nkelessis@doctors.org.uk postoperative radiotherapy and adjuvant chemotherapy. The neoplasm usually does not express oestrogen and progesterone receptors as well as Her2/neu protein. The tumour has a dismal prognosis with the size being a significant predictor on outcome.

Keywords Metaplastic $\cdot$ Breast carcinoma $\cdot$ Pathology Treatment

\section{Introduction}

Metaplastic carcinoma of the breast (MBC) accounts for less than $1 \%$ of all breast malignancies and tends to affect more frequently patients more than 40 years of age [1]. These tumours represent rare invasive carcinomas and are composed of epithelial and mesenchymal components. For historical purposes, alternative names included adenosquamous carcinoma, carcinosarcoma, matrix-producing carcinoma, sarcomatoid carcinoma, spindle cell carcinoma and squamous cell carcinoma [2, 3]. Mammographically, the tumour appearance varies, and it has been described as a wellcircumscribed, irregular or spiculated mass with or without microcalcifications $[4,5]$. The typical ultrasound appearance is that of a hypoechoic well-circumscribed lesion with a mixed echotexture depending on the constituent tissue subtypes contained within the mass [5]. The vast majority are high-grade tumours with hormone receptor-negative, overexpressing epidermal growth factor receptor (EGFR) but lack HER2/neu overexpression [6]. Approximately 70 $80 \%$ of metaplastic breast carcinomas overexpress the EGFR [7]. Symptomatology is not specific of the disease and mimics common invasive breast carcinomas. The tumour usually favours hematogenous metastases, although axillary node metastases are not uncommon. Fine needle aspiration 
biopsy (FNAB) is the first diagnostic tool. Neoadjuvant chemotherapy, surgery, radiotherapy and adjuvant chemotherapy as well as monoclonal antibodies against HER2 are applied as treatment options with various results. We herein present our experience with $\mathrm{MBC}$, with reference to the world literature.

\section{Report of the case}

A 59-year-old woman presented with a 7-month history of a large, painless tumour in the upper outer quadrant of her left breast. There was no past medical history or family history of breast cancer. On clinical examination, a palpable firm painless mass fixed to the overlying skin of the tail of the left breast was found. Gross lymphadenopathy in the axilla was also detected. There was no skin ulceration, nipple inversion or discharge. Mammography demonstrated a $6-\mathrm{cm}$ lobulated dense mass with irregular margins and enlarged lymph nodes in the left axilla (Fig. 1). Right breast was with normal findings. FNAB of the lesion was not relevant with metaplastic carcinoma because the material was inadequate. We did not perform a tru-cut biopsy and proceeded to excisional biopsy which demonstrated immunohistochemical and morphological characters compatible with low-differentiated

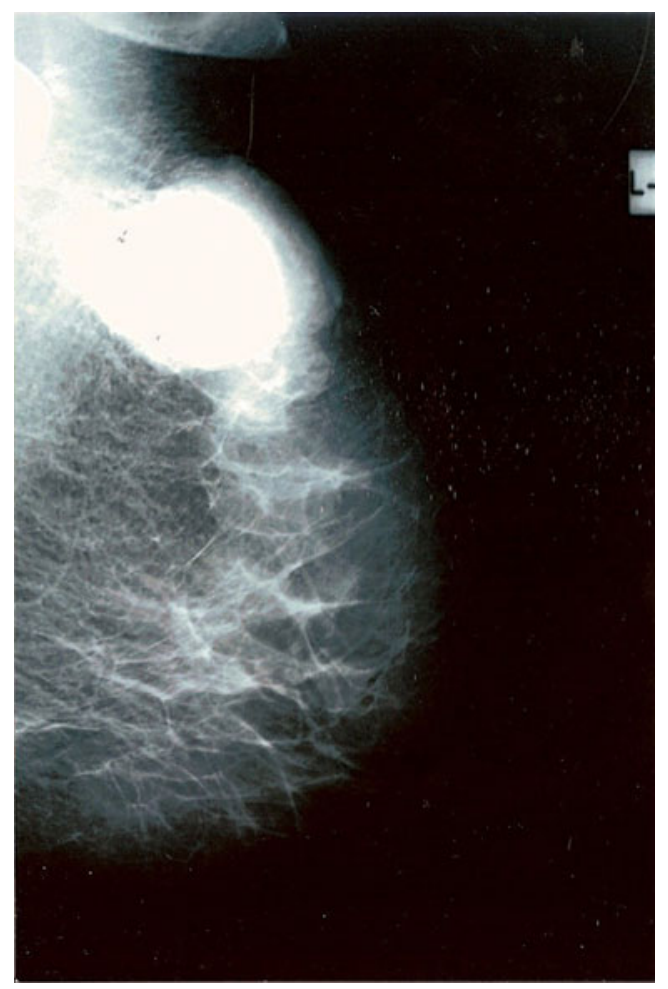

Fig. 1 Oblique mammogram of metaplastic breast carcinoma carcinoma. Then, a thorough evaluation followed. Blood tests including full blood count, erythrocyte sedimentation rate, liver function tests and tumour markers were all within normal limits. The chest $\mathrm{X}$-ray findings were normal. Chest CT scan demonstrated a solitary lesion located in the upper lobe of the right lung, measuring about $8 \mathrm{~mm}$ which was possibly of metastatic origin, whereas CT of the upper and lower abdomen were normal. Bone scan was normal as well. It was decided that the patient should receive neoadjuvant chemotherapy with epirubicin and taxanes. After three cycles, the patient denied to continue as this regimen was very poorly tolerated with no effect on the primary tumour. Then, we recommended a modified radical mastectomy, but the patient favoured a more conservative procedure, so she underwent a quadrantectomy with axillary clearance. Postoperative course was uneventful and the patient was discharged the next day with a drain placed in the left axilla. Pathology of the specimen revealed a $6.5 \mathrm{~cm}$ greatest diameter tumour with a large central cyst filled with $50 \mathrm{cl}$ of liquid. Microscopically, a welldifferentiated squamous component merges with a prominent spindle cell sarcomatoid component (Fig. 2). Immunohistochemically, the tumour cells showed strong positive reaction to cytokeratin 5.6 (Fig. 3). Oestrogen and progesterone receptors as well as c-erbB2 oncoprotein were negative. The tumour did not overexpress EGFR as well. Surgical borders were free of malignant infiltration, but metastatic deposits were found in 12 of 19 axillary lymph nodes. Six months following the operation, whilst she was on adjuvant chemotherapy, she developed osseous metastases in both femurs which were managed with radiotherapy. Consequently, the patient developed recurrence in the affected breast which was managed by total

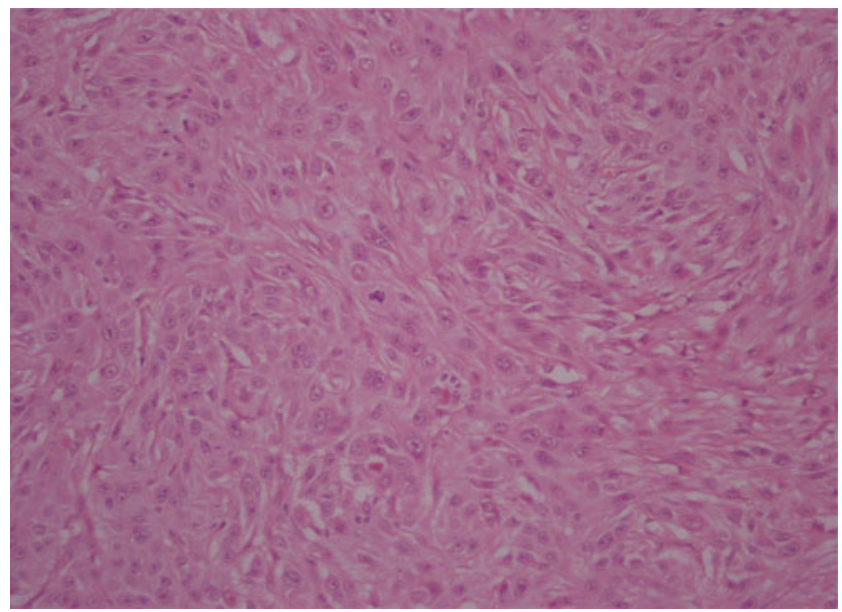

Fig. 2 Histological appearance of metaplastic carcinoma of the breast. There is a mixture of spindle and squamous cells. HE $\times 225$ 


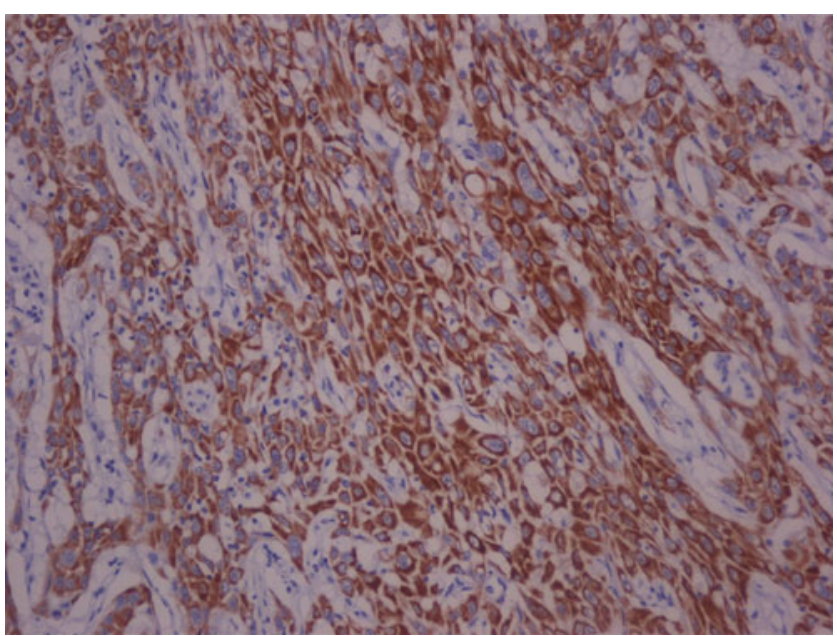

Fig. 3 Strong immunoreactivity of the carcinomatous cells to cytokeratine 5.6. Pap $\times 225$

mastectomy, and she finally died 4 months later from metastatic disease.

\section{Discussion}

The term metaplastic carcinoma, as a discrete entity, was first introduced by Hubos in 1973 [8].

Histologically, it is a poorly differentiated heterogeneous tumour containing ductal carcinoma cells admixed with areas of spindle, squamous, chondroid or osseous elements. The extent of metaplasia varies from microscopic foci to virtually complete replacement of the adenocarcinoma by the metaplastic elements. Regardless of the morphologic pattern, immunohistochemical and ultrastructural studies suggest that $\mathrm{MBC}$ are derived from multipotential undifferentiated cells $[9,10]$.

In the sarcomatoid type of metaplastic breast carcinoma, which was the variety encountered in our patient, there is a gradual transition from carcinomatous to sarcoma-like elements.

Triple negative tumours include a heterogeneous group of tumours with varying prognosis. The group with poor prognosis include metaplastic high-grade tumours discussed in the current case report. These tumours possess immunohistochemichal surrogate of basal-like cancer including expression of EGFR and positivity for CK5, CK6, CK14 and CK17.

Recently, a myoepithelial cell marker (p63) has been described in matrix-producing and metastatic breast cancer (MBC), suggesting that these tumours share a myoepithelial cell differentiation. This marker is highly sensitive and particularly specific for $\mathrm{MBC}$ with spindle and/or squamous areas and can be used when dilemmas in diagnostic workup between spindle cell MBC and other mesenchymal tumours appear [11].

In the last years, HER-2neu and human EGFR seem to attract the attention of the specialists due to the fact that humanised monoclonal antibodies against HER2 and therapies directed against the extracellular ligand-binding domain or the intracellular tyrosine kinase domain of EGFR have proven successful in treating certain types of human cancer. In breast carcinomas, EGFR expression is associated with a poor prognosis, although its prognostic value seems to be dependent on the oestrogen receptor status of the patient also $[7,12]$.

Axillary metastases in the sarcomatoid type are unusual, but it seems that in MBC, these occur in $25-30 \%$ of the cases $[13,14]$. Our patient presented with gross lymphadenopathy of the left axilla, and metastatic infiltration was detected in 12 of 19 lymph nodes. Metastases from MBC tend to be haematogenous rather than the more common route of the lymphatic spread. Pulmonary metastases are more common than brain, skeletal or hepatic [14]. Our patient presented with a solitary pulmonary metastasis of the right lung detected by chest CT scan, and 5 months after the operation, she developed osseous metastases in both thighs.

The current management of MBC is controversial due to the minimal amount of data and is based on information gathered from other breast malignancies.

Induction chemotherapy, surgery, hormonal therapy, adjuvant chemotherapy, radiotherapy, as well as new agents targeting the EGFR receptors, alone or in combination, consist the current armamentarium against this unusual disease.

Reviewing the world literature, the experience with neoadjuvant chemotherapy in $\mathrm{MBC}$ is extremely limited to evaluate its impact on the outcome [15].

Surgery remains the cornerstone of therapy in the majority of breast cancers. Until now, there are no standard guidelines for the treatment of MBC. Modified radical mastectomy (MRM), radical mastectomy, quadrantectomy or segmental mastectomy with or without axillary clearance have been applied as surgical alternatives. Because of the low risk of lymphatic spread, Caceres et al. [16] suggested that wide local excision with clear margins would be appropriate for local control. The higher incidence (53\%) of nodal metastases and larger median tumour size seen in his patients made him support the MRM as the optimal surgical treatment. Unlike invasive carcinoma of the breast, axillary lymph node (ALN) metastases in MBC do not correlate with clinical outcome [17, 18]. Other investigators have linked the risk of ALN metastases to the underlying histological subtype, being extremely low in spindle cell carcinoma [19]. 
Systemic therapy also appears to be less effective. According to some studies, early relapses and no response to adjuvant chemotherapy were demonstrated $[20,21]$.

Breast conservation with adjuvant radiation may be a reasonable option for metaplastic breast carcinoma. In clear surgical margins $(2 \mathrm{~cm})$, post-mastectomy radiation is not recommended; otherwise, radiation is indicated [7]. It is of interest that our patient developed recurrence in the affected breast, although she had received postoperative radiotherapy and the pathologist confirmed clear resection margins.

Patients with MBC, particularly those with metastatic disease, could be appropriate candidates for innovative therapeutic regimens.

Adjuvant endocrine treatment or Her-2 targeted treatment with a monoclonal antibody (trastuzumab) is usually not recommended in the majority of $\mathrm{MBC}$ due to negative oestrogen and progesterone receptors as well as negative Her-2 overexpression [22]. The frequent expression of EGFRs in the absence of steroid receptors or other receptors of the EGFR family might render metaplastic breast carcinomas even more sensitive to EGFR tyrosine kinase inhibitors [22]. In breast carcinomas, EGFR expression is associated with a poor prognosis, although its prognostic value seems to be dependent on the oestrogen receptor status of the patient also [23]. New treatment opportunities may exist with the development of agents targeting the EGFR receptor such as gefitinib and cetuximab. In a study of 20 cases of MBCs, 14 of 20 were positive for EGFR expression, supporting the utility of such targeted therapies [22]. Further research is needed to fully evaluate the potential of such therapy in patients with MBC.

Although there are no sufficient data to determine the overall prognosis, survival is highly dependent on tumour size, histologic type, grade and lymph node involvement. Compared to adenocarcinomas, MBC appears a more aggressive behaviour and worse prognosis, and the overall 5 -year survival rate is approximately $40 \%$ [24]. The prognosis is poorer in sarcomatoid variety of metaplastic carcinoma than in the other types, and the metastatic potential tends to be haematogenous rather than the more common route of the lymph node spread.

In conclusion, this rare malignancy of the breast demonstrates an aggressive behaviour with dismal prognosis. The majority of these tumours is oestrogen- and progesterone receptor-negative, HER2-neu-negative, but usually overexpresses EGFR. The tumour does not favour the axillary nodes but tends to recur locally and frequently metastasizes to the lung. The small number of patients in the majority of the studies does not allow safe conclusions regarding the optimal treatment. Prognosis is variable and highly dependent on tumour's size, grade and lymph node involvement. Nowadays, surgeons, medical oncologists and radiotherapists who are involved in the management of this disease still rely on data from other types of breast cancer. A better understanding of tumour biology and pathology in conjunction with more innovated agents and clinical trials will lead to optimal treatment of this uncommon disease.

Conflict of interest There is no actual or potential conflict of interest in relation to this article.

\section{References}

1. Rosen PP (1997) Rosen's breast pathology. Lippincott-Raven, Philadelphia, pp 375-395

2. Wargotz ES, Norris HJ (1989) Metaplastic carcinoma of the breast. I. Matrix-producing carcinoma. Human Pathol 20:628-635

3. Eggers JW, Chesney TM (1984) Squamous cell carcinoma of the breast: a clinicopathologic analysis of eight cases and review of the literature. Hum Pathol 15:526-531

4. Brenner RJ, Turner RR, Schiller V, Arndt RD, Giuliano A (1998) Metaplastic carcinoma of the breast: report of three cases. Cancer 82:1082-1087

5. Park JM, Han BK, Moon WK, Choe YH, Ahn SH, Gong G (2000) Metaplastic carcinoma of the breast: mammographic and sonographic findings. J Clin Ultrasound 28:179-186

6. Bellino R, Arisio R, D'Addato F, Attini R, Durando A, Danese S et al (2003) Metaplastic breast carcinoma: pathology and clinical outcome. Anticancer Res 23:669-673

7. Rosen PP, Ernsberger D (1987) Low grade adenosquamous carcinoma. A variant of metaplastic mammary carcinoma. Am J Surg Pathol 115:351_/8

8. Huvos AG, Lucas JC, Foote FW (1973) Metaplastic breast carcinoma: rare form of mammary cancer. NY State J Med 73:1078-1082

9. Carter CL, Allen C, Henson DE (1969) Relation of tumor size, lymph node status and survival in 24,740 breast cancer cases. Cancer 63:181-187

10. Harris JR, Lippman ME, Morrow M, Osborne CK (2004) Diseases of the breast, 3rd edn. Lippincott Williams \& Wilkins, Philadelphia, pp 557-558

11. Koker MM, Kleer CG (2004) p63 expression in breast cancer: a highly sensitive and specific marker of metaplastic carcinoma. Am J Surg Pathol 28:1506-1512

12. Hynes NE, Lane HA (2005) ERBB receptors and cancer: the complexity of targeted inhibitors. Nat Rev Cancer 5:341-354

13. Oberman HA (1987) Metaplastic carcinoma of the breast. Am J Surg Pathol 11:918-929

14. Pitts WC, Rojas VA, Gaffey MJ, Rouse RV, Esteban J (1991) Carcinomas with metaplasia and sarcomas of the breast. Am J Clin Pathol 95:623-632

15. Adher D, Sayed Al, Amr N, Weshy El, Tulbach A, Rachal M (2006) Metaplastic carcinoma of the breast clinical presentation, treatment results and prognostic factors. Acta Oncol 45:188-195

16. Caceres M, Shih J, Eckert M, Gardner R (2002) Metaplastic carcinoma in an ectopic breast. South Med J 95:462-466

17. Paterson DA (1992) Carcinosarcoma and metaplastic carcinomas. The Breast 1:136-137

18. Gutman H, Pollock RE, Janjan NA, Johnston DA (1995) Biologic distinctions and therapeutic implications of sarcomatoid metapla- 
sia of epithelial carcinoma of the breast. J Am Coll Surg 180:193199

19. Bauer TW, Rostock RA, Eggleston JC, Baral E (1984) Spindle cell carcinoma of the breast: four cases and review of the literature. Hum Pathol 15:145-152

20. Rayson D, Adjei AA, Suman VJ, Wold LE, Ingle J (1999) Metaplastic breast cancer: prognosis and response to systemic therapy. Ann Oncol 10:413-419

21. Choa TC, Wang CS, Chen SC, Chen MF (1999) Metaplastic carcinoma of the breast. J Surg Oncol 71:220-225

22. Leibl S, Moinfar F (2005) Metaplastic breast carcinomas are negative for Her-2 but frequently express EGFR (Her-1): potential relevance to adjuvant treatment with EGFR tyrosine kinase inhibitors? J Clin Pathol 58(7):700-704

23. Tsutsui S, Ohno S, Murakami S, Hachitanda Y, Oda S (2002) Prognostic value of epidermal growth factor receptor (EGFR) and its relationship to the estrogen receptor status in 1029 patients with breast cancer. Breast Cancer Res Treat 71:6775

24. Luini A, Aquilar M, Gatti G, Fasani R, Botteri E, Brito JA et al (2007) Metaplastic carcinoma of the breast, an unusual disease with worse prognosis: the experience of the European Institute of Oncology and review of the literature. Breast Cancer Res Treat 101(3):349-353 